\title{
Influência de 12 anos de prática de atividade física regular em programa supervisionado para idosos
}

\author{
Influence of a 12-year supervised physical activity program \\ for the elderly
}

Resumo - O envelhecimento é um processo inevitável, associado com o declínio fisiológico e da capacidade funcional do ser humano. O objetivo deste estudo foi verificar os efeitos de 12 anos de prática de atividade física em programa supervisionado, sobre a aptidão funcional em idosos. Dez idosas (idade média de 65 anos) participaram do estudo. Elas foram divididas em dois grupos: a) grupo treinado (GT), composto de 5 participantes que estavam atendendo um programa supervisionado que incluía diferentes tipos de atividades físicas de intensidade moderada; b) grupo não treinado (GNT), participantes que não estavam engajadas em programa supervisionado de atividade física, ambos nos últimos 12 anos. A aptidão funcional foi avaliada por meio da bateria de testes para idosos da AAHPERD que é composta por cinco testes: coordenação, flexibilidade, resistência de força, agilidade e equilíbrio dinâmico e resistência aeróbia geral. Os resultados mostram que as idosas que permaneceram no programa de atividade física apresentaram melhor desempenho nos testes motores. Portanto, enquanto idosas que continuam a participar regularmente de atividades físicas num programa supervisionado tendem a melhorar ou manter todos os componentes de aptidão funcional, mesmo durante o longo período de 12 anos, aquelas que só realizam atividades físicas requeridas nas tarefas da vida diária, tendem a reduzir a maioria daqueles componentes. As evidências do presente estudo possibilitam predizer diferenças de aptidão funcional, cada vez maiores, entre os dois grupos, com o passar do tempo, o que pode produzir efeitos de direção oposta nas respectivas qualidades de vida.

Palavras-chave: Envelhecimento; Aptidão funcional; Atividade física; Qualidade de vida.

1 Universidade Federal de São Paulo, Faculdade de Educação Física, Modalidade Saúde, Departamento de Biociências, Santos, SP. Brasil.

2 Universidade Estadual Paulista, Faculdade de Educação Física, Departamento de Biodinâmica, Rio Claro, SP. Brasil.

Recebido em 11/05/08 Revisado em 17/08/08 Aprovado em 12/09/08
Abstract - Aging is an inevitable process and is associated with declining physiological and functional capacity in humans. The objective of this study was to determine the effects of a 12-year supervised physical training program on functional fitness in the elderly. Ten women (mean age: 65 years) participated in the study. The subjects were divided into two groups: a) a trained group consisting of women who had been attending a supervised program including different types of physical activities of moderate intensity over the last 12 years; b) an untrained group consisting of women who were not engaged in any supervised physical activity program over the last 12 years. Functional fitness was assessed using the AAHPERD field-test battery which comprises five single motor tests: coordination, flexibility, strength endurance, agility and dynamic balance, and overall aerobic endurance. The results showed a better performance of elderly women who participated in a physical activity program over the last 12 years. Thus, whereas elderly women who perform regular physical activities in a supervised program tend to show improvement of all functional fitness components even after a period of 12 years, a tendency towards a reduction in most of these components is observed in their non-active peers. These findings seem to predict an increasing gap in functional fitness between these two groups as they grow older, with opposite effects on the quality of life of these subjects.

Key words: Aging; Functional fitness; Physical activity; Quality of life. 


\section{INTRODUÇÃO}

Uma das conseqüências associadas ao processo de envelhecimento é a diminuição da aptidão funcional e consequentemente, da capacidade de realizar as atividades da vida diária sem necessitar de auxilio de outras pessoas ou até mesmo de artefatos como bengalas, andadores, cadeira de rodas, etc. Diversos estudos mostram que o aumento do sedentarismo, com o envelhecimento, é um dos fatores cruciais do declínio da capacidade funcional do ser humano, e que a prática regular de atividade física propicia reversibilidade parcial ${ }^{1}$, manutenção ${ }^{2}$, ou redução da taxa de declínio da mesma ${ }^{3}$. Isto, felizmente, indica que o organismo envelhecido retém a treinabilidade ${ }^{4,5}$.

No entanto, apesar de ter aumentado o interesse por estudos relacionando à aptidão funcional e ao envelhecimento, especialmente preocupado com a manutenção da capacidade funcional, já que a prática de atividade física tem sido evidenciada como um caminho capaz de retardar uma série de morbidades e mortalidades em grupos nesta faixa etária, ainda há muito que ser pesquisado, principalmente, a necessidade de estudos longitudinais com períodos prolongados de tempo, haja vista que a esmagadora maioria dos estudos tem sido realizada pelo máximo de 1 ano de protocolo de treinamento e, mais frequentemente, de 3 a 6 meses ${ }^{6}$. Tal diagnóstico se faz importante para que os programas de atividade física voltados a pessoas idosas sejam valorizados, bem como o número destes e dos participantes no país aumentado, com o intuito de contribuir não só com a qualidade de vida das pessoas, mas também, aumentando a perspectiva de uma vida ativa.

Neste sentido, o presente estudo tem como objetivo verificar se um programa de atividade física regular, composto de atividades físicas generalizadas e com intensidade moderada, desenvolvido durante 12 anos, tem proporcionado benefícios à aptidão funcional de idosos.

\section{PROCEDIMENTOS METODOLÓGICOS}

\section{Amostra}

Participaram deste estudo 10 voluntárias divididas em 2 grupos: GNT ( $n=5$ ), composto por idosas (idade média de 64,2 anos), que realizavam atividades da vida diária independentemente e haviam participado de um programa supervisionado de atividade física, mas que, nos últimos doze anos, não fizeram parte de nenhum programa supervisionado de ati- vidade física e; GT (n=5) com idade média de 65,8 anos, com as mesmas características do GNT, mas continuaram a participar de um programa de atividade física supervisionado, que incluía atividades generalizadas (dança, exercícios resistidos, esportes adaptados, ginástica aeróbia, atividades recreativas e outras), de intensidade moderada; com sessões de 1 hora de duração, três vezes por semana, durante 9 meses por ano, nos últimos 12 anos.

\section{Procedimentos}

Todos os participantes assinaram um termo de consentimento de participação no estudo, permitindo publicação dos resultados obtidos. Antes de realizarem o protocolo de avaliação, foram examinados previamente por médico, cujas informações de saúde foram encaminhadas ao profissional de educação física pesquisador. $O$ projeto de pesquisa foi enviado e aprovado pela comissão de ética da Universidade Estadual Paulista-UNESP - Campus de Rio Claro-SP.

Os critérios de inclusão da amostra foram os seguintes: ter idade superior a 60 anos; ser do sexo feminino, devido ao fato dos valores normativos da aptidão funcional geral existente no Brasil, avaliados por meio da bateria de testes da AAHPERD, terem sido desenvolvidos, até o momento, somente para o sexo feminino, por Zago e Gobbi; realização de todos os testes da bateria; não apresentar alterações osteomusculares limitantes à realização dos testes e que aceitaram participar voluntariamente da pesquisa. Não atendiam a estes critérios, 4 indivíduos do GT e 5 indivíduos do GNT, que então, não participaram do estudo.

\section{Protocolo de avaliação}

Os instrumentos utilizados foram: a bateria de testes para idosos da American Alliance for Health, Physical Education, Recreation and Dance - AAHPERD ${ }^{8}$, que apresenta testes motores que avaliam a coordenação $(\mathrm{COO})$, resistência de força (RF), flexibilidade (FLEX), agilidade e equilíbrio dinâmico (AED), e a resistência aeróbia geral (RAG), ou seja, a aptidão funcional geral dos idosos. Nessa bateria, coordenação, agilidade, equilíbrio dinâmico e resistência aeróbia geral são mensurados em segundos, flexibilidade é medida em centímetros, resistência de força é o número de repetições que o idoso consegue realizar, flexionando o braço com o peso, em 30 segundos e o IAFG é a soma dos índices percentis dos 5 testes. A escolha da bateria da AAHPERD se deve aos seguintes aspectos: é considerada a mais antiga bateria de teste utilizada 
para avaliar a aptidão funcional dos idosos ${ }^{9}$, de fácil aplicação e montagem, baixo custo, não requer grandes espaços, a existência de valores normativos para idosos brasileiros do sexo feminino, ${ }^{7,9}$, e relacionamento dos resultados com o desempenho funcional nas atividades diárias dos idosos.

\section{Estatística}

Para classificar, qualitativamente, os resultados dos testes motores isoladamente e o Índice de Aptidão Funcional Geral (IAFG), foram utilizadas as tabelas com os valores normativos, baseados em escores percentis, para mulheres de 60-70 anos, desenvolvidas por Zago e Gobbi?. Tais dados oferecem uma visão de como os grupos se encontravam no início e após 12 anos de prática ou não do programa de atividade física e proporcionam uma análise qualitativa da aptidão funcional do idoso, comparada com a população de mesmo gênero e idade. Para a comparação das médias inter-grupos dos resultados dos testes motores foi utilizada a ANOVA $(p<0,05)$.

\section{RESULTADOS}

As características de idade e medidas antropométricas das idosas constam na Tabela 1. Estes dados trazem informações tanto das praticantes quanto daquelas que deixaram de participar do programa de atividade física regular supervisionada no período de 12 anos.

Os resultados dos 5 testes motores das idosas aplicados no início e após 12 anos de prática ou não do programa de atividade física foram agrupados na Tabela 2. Os resultados mostram que o grupo que continuou a praticar exercícios sob orientação do profissional de educação física apresentou manutenção ou melhoras no desempenho motor, e as idosas que deixaram o programa tiveram declínio em quase todos os testes aplicados.

A classificação dos resultados dos componentes de aptidão funcional isoladamente e do IAFG estão representados na Tabela 3. Observa-se que as idosas que permaneceram no programa ao longo dos 12 anos apresentam melhores resultados nos testes motores, e isso, também refletiu no IAFG que se apresenta com um índice classificatório muito bom, enquanto o grupo que deixou o programa apresenta piores resultados e consequentemente, índice apenas regular.

Tabela 1. Características de idade e antropométricas das participantes do grupo não treinado (GNT) e grupo treinado (GT) ao final de 12 anos de estudo.

\begin{tabular}{lcc}
\hline Variáveis & $\begin{array}{c}\text { GNT } \\
(\mathrm{n}=5)\end{array}$ & $\begin{array}{c}\text { GT } \\
(\mathrm{n}=5)\end{array}$ \\
\hline Idade (anos) & $65,60 \pm 4,03$ & $64,20 \pm 3,15$ \\
Massa Corporal $(\mathrm{Kg})$ & $70,60 \pm 13,3$ & $66,10 \pm 13,7$ \\
Estatura $(\mathrm{m})$ & $1,59 \pm 0,05$ & $1,57 \pm 0,03$ \\
IMC $\left(\mathrm{Kg} / \mathrm{cm}^{2}\right)$ & $27,80 \pm 5,00$ & $26,60 \pm 5,30$ \\
\hline
\end{tabular}

Tabela 2. Resultados de testes motores de aptidão funcional em mulheres participantes (GT) e não participantes de programa de atividades físicas (GNT).

\begin{tabular}{lcccc}
\hline \multirow{2}{*}{$\begin{array}{l}\text { Grupos } \\
\text { Variáveis }\end{array}$} & \multicolumn{2}{c}{ GNT $(n=5)$} & \multicolumn{2}{c}{ GT $(n=5)$} \\
\cline { 2 - 5 } & pré & Pós & pré & Pós \\
\hline COO (s) & $11,7 \pm 2,5$ & $11,9 \pm 1,5$ & $12,8 \pm 3,5$ & $9,2 \pm 2^{*}$ \\
FLEX (cm) & $57,15 \pm 4,0$ & $57,4 \pm 3,2$ & $67 \pm 7,5^{\text {b }}$ & $71,5 \pm 5^{*}$ \\
AED (s) & $19,47 \pm 5,5$ & $22,84 \pm 2,7$ & $21,6 \pm 2,8$ & $19,4 \pm 1,8^{*}$ \\
RF (rep.) & $23,4 \pm 6,9$ & $23,8 \pm 2,0$ & $24,4 \pm 8,0$ & $28,2 \pm 5,2$ \\
RAG (s) & $474,6 \pm 31,8$ & $529,6 \pm 58,3$ & $464,4 \pm 45,4$ & $460,2 \pm 37,1$ \\
\hline
\end{tabular}

* $=\mathrm{p}<0,05$ (GT \# GNT) no mesmo ano, COO = Coordenação, FLEX $=$ Flexibilidade, $\mathrm{AED}=$ Agilidade e Equilíbrio Dinâmico $\mathrm{RF}=$ Resistência de Força, RAG = Resistência aeróbia geral.

Tabela 3. Classificação dos resultados dos testes motores isoladamente e do Índice de Aptidão Funcional Geral (IAFG) de idosas praticantes (GT) e não praticantes (GNT) de programa supervisionado de atividade física, por um período de 12 anos. (Baseada nas tabelas de valores normativas de ZAGO e GOBBI) ${ }^{7}$.

\begin{tabular}{|c|c|c|c|c|}
\hline \multirow{2}{*}{$\begin{array}{l}\text { Grupos } \\
\text { Variáveis }\end{array}$} & \multicolumn{2}{|c|}{$\begin{array}{c}\text { GNT }(n=5) \\
\text { Pontos percentis - Conceito }\end{array}$} & \multicolumn{2}{|c|}{$\begin{array}{c}\text { GT }(n=5) \\
\text { Pontos percentis - Conceito }\end{array}$} \\
\hline & pré & pós & pré & Pós \\
\hline $\mathrm{COO}$ & 57 - Regular & 51 - Regular & 39 - Fraco & 88 - Muito Bom \\
\hline FLEX & 71 - Bom & 71 - Bom & 91- Muito Bom & 95 - Muito Bom \\
\hline AED & 82 - Muito Bom & 44 - Regular & 57 - Regular & 83 - Muito Bom \\
\hline RF & 51 - Regular & $70-$ Bom & 59 - Regular & 88 - Muito Bom \\
\hline RAG & $70-$ Bom & 22 - Fraco & $78-$ Bom & 80 - Muito Bom \\
\hline IAFG & 331 - Bom & 258 - Regular & $324-$ Bom & 434 - Muito Bom \\
\hline
\end{tabular}

$\mathrm{COO}=$ Coordenação, FLEX = Flexibilidade, $\mathrm{AED}=$ Agilidade e Equilíbrio Dinâmico, RF $=$ Resistência de Força, RAG $=$ Resistência aeróbia geral. 


\section{DISCUSSÃO}

Os resultados obtidos em nosso estudo são condizentes a outros previamente realizados e que utilizaram a mesma bateria de testes da AAHPERD $1,10-14$. $\mathrm{O}$ aspecto mais interessante da comparação com outros trabalhos é a constatação de que, apesar do longo período de tempo abrangido pelo presente estudo, as participantes do GT continuam a se beneficiar da prática regular de atividade física, ou seja, os ganhos obtidos por períodos de treinamento de próximos a 1 ano são mantidos ou mesmo melhorados. Isto leva a assumir que o programa de atividade física supervisionado que o GT participou, por 12 anos, não objetiva desempenho máximo, mas sim um nível adequado de capacidade funcional que, quando obtido, não necessita de sobrecarga de esforços para manter tal nível, bastando continuar a prática com a mesma duração de sessões, frequência semanal e intensidade. Em outras palavras, as atividades do programa podem representar uma sobrecarga de trabalho apenas no início do atendimento do programa, o organismo, então, adapta-se a esta sobrecarga e permanece num nível adequado de capacidade funcional por longo período de tempo.

Analisando, ainda, a Tabela 2, no início do programa, apenas FLEX se mostrou diferente, tendo o GT apresentado melhor desempenho comparado ao GNT. Já, comparando os grupos após 12 anos, o GT apresenta significativamente melhores resultados de COO, FLEX e AED e tendência de superioridade em RF e RAG. Outro achado relevante é que, após 12 anos, o GNT apresenta tendência de declínio em três (COO, RAG, AED) dentre os cinco componentes da aptidão funcional avaliados e, interessantemente, manutenção de um (FLEX) e de melhora em outro (RF). Isto leva à interpretação de que apenas a realização de atividades da vida diária independentemente, como é o caso das participantes do GNT ,não representa estímulo suficiente para se contrapor ao declínio associado ao envelhecimento nesse período.

Assim, se o processo natural de envelhecimento leva a um declínio funcional do idoso ${ }^{15}$, o simples fato de ter havido manutenção, ou tendência para melhora dos níveis dos componentes da aptidão funcional, ao longo do período de 12 anos, no GT, já representa um saldo positivo para aptidão funcional dos participantes.

No nível de AED, o declínio da força poderia representar menor desempenho, enquanto, no teste de andar, um declínio no $\mathrm{VO}_{2}$ max poderia talvez limitar a execução, haja vista que, um menor $\mathrm{VO}_{2}$ max poderia exigir um esforço, no teste, numa maior percentagem daquele $\mathrm{VO}_{2}$ max. Macedo et al. ${ }^{16}$ mostraram que mulheres nas faixas etárias de 60-69 e de 70-81, participantes regulares de atividade física apresentam maiores índices de $\mathrm{VO}_{2}$ max, quando comparadas com mulheres da mesma faixa etárias inativas fisicamente. Desta forma, apesar de inevitável, o declínio na potência aeróbia, com o envelhecimento, é menor nos idosos treinados, mesmo com exercícios de baixas intensidades.

Benedetti e Petroski ${ }^{17}$ encontraram uma melhora das variáveis da aptidão funcional com um programa de exercícios físicos, principalmente, nas variáveis de força muscular, o que contribuiu na velocidade de andar e no equilíbrio. Assim sendo, levando em consideração que a mobilidade é uma variável extremamente importante, associada não somente com a qualidade de vida para indivíduos idosos, mas também com a longevidade, podemos considerar que um programa supervisionado de atividade física pode beneficiar seus participantes, haja vista que evidências científicas apontam para o fato de que o envelhecimento promove declínio na velocidade de andar em homens e mulheres ${ }^{17,18-21}$.

Além disso, quando comparamos ambos os grupos após os 12 anos, o grupo GNT apresenta, também, valores significativamente menores nos testes de coordenação e flexibilidade, o que demonstra que a atividade física desenvolvida num programa de atividade física supervisionado é positiva para outras capacidades físicas.

Entretanto, a manutenção dos níveis de flexibilidade para o GNT pode ser explicada pelo fato de que o declínio de tal capacidade física ocorre em diferentes taxas com o avanço da idade, havendo uma grande variabilidade interindividual, como já observado por Ueno et al..$^{22}$ Isso faz com que indivíduos numa mesma faixa etária possam apresentar grau de flexibilidade do quadril diferente, e talvez, para os sujeitos do GNT, o período de 12 anos ainda não tenha sido suficiente para promover significantes declínios e no caso da flexibilidade, apenas as atividades de vida diária tenham sido suficientes para a manutenção do nível de tal componente da aptidão funcional.

Contudo, é essencial ressaltar que, independentemente de existir variabilidade quanto ao grau de declínio, com o avançar da idade, a realização de exercícios pode ser considerado um elemento importante para manutenção e ou aumento da flexibilidade. Os dados neste estudo evidenciam esta hipótese, já que o grupo GT apresenta níveis 
superiores no teste de flexibilidade após 12 anos, confirmando o efeito positivo da atividade física para esse componente.

Adicionalmente, quando analisamos os dados referentes aos testes de resistência de força de membro superior (bíceps), verificou-se que o GT obteve um número de repetições superior após o período de 12 anos de prática do programa generalizado de exercícios quando comparado ao GNT. No entanto, o fato de ambos os grupos não apresentarem declínio na RF, foi uma grande surpresa.

Uma possível explicação para a o fato de não ter ocorrido declínio de RF, no período de 12 anos, principalmente, no GNT, pode ser devido a alguns fatores: 1) via de regra, com o envelhecimento, ocorre menor declínio de membros superiores, quando comparados com os membros inferiores ${ }^{23,24}$;2) existem estudos mostrando que pessoas que realizam as atividades domésticas do dia-dia independentemente, as quais, muitas vezes, requerem resistência de força de membros superiores (limpar a casa, lavar roupa, carregar sacolas de compra, etc.) podem prevenir o declínio nesta capacidade física e isto explicaria a manutenção de força no $\mathrm{GNT}^{18,25}$. 3) é possível que as idosas do GT pelo fato de não realizarem exercícios regulares supervisionados anteriores ao ingresso no programa supervisionado, seja a explicação do baixo rendimento na primeira avaliação, e a participação regular ao longo dos anos propiciou uma melhora significativa nessa capacidade.

Contudo, é importante ressaltar que, comparando-se os resultados inter-grupos, ao final do estudo, verifica-se que os indivíduos do GT apresentam melhor desempenho de RF comparados com os de GNT. Isto indica que a aderência à prática regular de atividade física, num programa supervisionado, é bastante benéfica também para a capacidade de RF.

Assim, um maior declínio das capacidades relacionadas à extremidade inferior (AED e RAG) no GNT e manutenção das mesmas, com tendência a melhoras no grupo GT, pode ser devido a uma ausência de uniformidade, que inclui magnitudes de declínio de força diferenciadas entre segmentos corporais, pois há evidências de um maior efeito do envelhecimento, na redução da força dos músculos dos membros inferiores comparada com a de membros superiores $^{23,26}$. Por isso, o fato do GNT ter apresentado declínio, após 12 anos, nas capacidades relacionadas ao membro inferior (AED e RAG) está de acordo com os estudos encontrados na literatura.

Além disso, ao analisar o IAFG, podemos observar que os idosos do GT melhoraram, passando do nível bom para o nível muito bom de aptidão funcional geral, enquanto que o GNT piorou, passando do nível bom para o regular. Isto fortalece a evidência, mostrada neste estudo, de que as práticas de atividades, num programa supervisionado, contribuíram para a melhora ou prevenção de declínio na aptidão funcional ao longo de 12 anos.

Assim, o presente estudo mostrou que não só componentes específicos, mas também a aptidão funcional geral de idosos pode ser beneficiada pela participação em um programa supervisionado de atividades generalizadas de intensidade moderada. Ainda mais relevante é que tais benefícios são mantidos por mais de uma década. Em recentes considerações, Peterson et al. ${ }^{27}$ sugerem que um programa de atividade física variado, com exercícios de força, resistência aeróbia, agilidade entre outros, de intensidade moderada a vigoroso, pode minimizar o declínio da aptidão funcional, distanciando o idoso do limiar de incapacidade e dependência.

Por outro lado e de maneira coerente, este estudo também mostra que idosas que restringem seus níveis de atividade física àqueles requeridos para o desempenho independente de atividades de vida diária, tendem a ter seus níveis de componentes e a própria aptidão funcional como um todo, reduzidos a médio e longo prazo.

Assim, apesar das limitações relacionadas ao tamanho da amostra e da mesma ser constituída apenas por mulheres, nossos resultados são relevantes, pois se trata de um estudo longitudinal com idosas brasileiras, e dados científicos desta natureza são escassos, o que dificulta uma análise positiva ou negativa de programas de atividades físicas direcionados ao público idoso sobre a aptidão funcional e qualidade de vida.

\section{CONCLUSÃO}

Se levarmos em consideração que ocorre um declínio acentuado da aptidão funcional, principalmente, a partir dos 65 anos de idade, como mostra o estudo de Demura et al..$^{15}$, e os nossos participantes se encontram nessa faixa etária, o engajamento ou a continuação na prática regular de atividade física num programa supervisionado torna-se ainda mais importante, nos próximos anos. Tal fato permite predizer, com o passar dos anos, uma amplitude cada vez maior de diferença de aptidão funcional entre o grupo de idosas que restringem seus níveis de atividade física às requeridas pelas tarefas da vida diária e aquele que acrescenta a participação regular em um programa supervisionado de atividades 
físicas variadas, de intensidade moderada, realizado três vezes por semana e com sessões de uma hora de duração. Como consequência, este comportamento diferenciado da aptidão funcional nos citados grupos pode interferir com as respectivas qualidades de vida em direções opostas.

\section{REFERÊNCIAS BIBLIOGRÁFICAS}

1. Ferreira L, Gobbi S. Agilidade geral e agilidade de membros superiores em mulheres de terceira idade treinadas e não treinadas. Rev Bras Cineantropom Desempenho Hum 2003;5(1):46-53.

2. Berk DR, Hubert HB, Fries JS. Associations of change in exercise level with subsequent disability among seniors: a 16-year longitudinal study. J Gerontol A Biol Sci Med Sci 2006;61(1):97-102.

3. Brach JS, Simonsick EM, Kritchevsky S, Yaffe K, Newman $A B$. The association between physical functional and lifestyle activity and exercise in the healthy, aging and body composition study. J Am Geriatr Soc 2004;52(4):502-509.

4. Pauli JR, Souza LS, Gobbi S, Zago AS. Efeitos de um programa de treinamento físico personalizado sobre a aptidão funcional, composição corporal e bioquímica sanguínea em idosas. Motricidade 2005;1(2):116-125.

5. Bruce B, Fries JF, Hubert H. Regular vigorous physical activity and disability development in healthy overweight and normal-weight seniors: 13-year study. Am J Public Health 2008;98(7):1294-1299.

6. Gobbi S. O paradoxo do enfrentamento dos benefícios do envelhecimento populacional: o papel da atividade física e das ciências da motricidade humana. Rev Bras Ativ Física Saúde 2003;8(3):1.

7. Zago AS, Gobbi S. Valores normativos da aptidão funcional de mulheres de 60 a 70 anos. Rev Bras Ciênc Mov 2002;11(2):77-86

8. Osness WH. Functional fitness assessment for adults over 60 years. The American Alliance For Health, Physical Education, Recreation and Dance. Reston: American Alliance for Health, Physical Education, Recreation and Dance; 1990.

9. Benedetti TRB, Mazo GZ, Gobbi S, Amorim M, Gobbi LTB, Ferreira L, et al. Valores Normativos de Aptidão Funcional em mulheres de 70 a 79 anos. Rev Bras Cineantropom Desempenho Hum 2007;9(1):28-36.

10. Villar R, Zago AS, Polastri PF, Miyasike VS, Gobbi S. Efeito de um programa de atividades físicas generalizadas e de intensidade moderada na resistência aeróbia em idosos. Motriz 2001;7(1):s121.

11. Zago AS, Polastri PF, Villar V, Silva VM, Gobbi S. Efeito de um programa geral de atividade física de intensidade moderada sobre os níveis de resistência de força em pessoas da terceira idade. Rev Bras Ativ Física Saúde 2000;5(3):42-51.

12. Polastri PF, Silva VM, Villar R, Zago AS, Gobbi S. Alterações nos níveis de coordenação de pessoas da terceira idade através de um programa de atividade física generalizada. Motriz 1999;5(1):115.

13. Petroski EC. Efeito de um programa de atividade física na terceira idade. Rev Bras Ativ Física Saúde 1997;2(2):34-40.
14. Rosa MF, Mazo GZ, Da Silva AH, Brust C. Efeito do período de interrupção de atividades aquáticas na aptidão funcional de idosas. Rev Bras Cineantropom Desempenho Hum 2008;10(3):237-242.

15. Demura S, Minami M, Nagasawa Y, Tada N, Matasuzawa J, Sato S. Physical-Fitness declines in older Japanese adults. J Aging Phys Act 2003;11:112-122.

16. Macedo IF, Duarte CR, Matsudo VKR. Avaliação da potência aeróbica em adultos de diferentes idades. Rev Bras Ciênc e Mov 1987;1(1):7-13.

17. Benedetti TRB, Petroski EL. Idosos asilados e a pratica de atividade física. Rev Bras Ativ Física Saúde 1999;4(3):5-16.

18. Rantanen T, Heikkinen E. The role of habitual physical activity in preserving muscle strength from age 80 to 85 years. J Aging Phys Act 1998;6:121-132.

19. Daley M, Spinks W. Exercise, mobility and aging. Sports Med 2000;29(1):1-12.

20. Hirvensalo M, Rantanen T, Heikkinen E. Mobility difficulties and physical activity as predictors of mortality and loss of independence in the community-living older population. J Am Geriatr Soc 2000;48(5):493-498.

21. Kalapotharakos V, Smilios L, Parlavatzas A, Tokinakidis SP. The effect of moderate resistance training and detraining on muscle strength and power in older men. J Geriatr Phys Ther 2007;30(3):109-113.

22. Ueno LM, Okuma SS, Miranda ML, Filho WJ. Análise dos efeitos quantitativos e qualitativos de um programa de educação física sobre a flexibilidade do quadril em indivíduos com mais de 60 anos. Motriz 2000;6(1):9-16.

23. Lynch NA, Metter EJ, Lindle RS, Fozard JL, Tobin JD, Roy TA. Muscle quality. Age-associated differences between arm and leg muscle groups. J Appl Physiol 1999;86(1): 188-194.

24. Nikolic M, Malmar-Dragojevic D, Bobinac D, Bajek S, Jerkovic R, Soicvranic T. Age-related skeletal muscle atrophy in humans: an immunohistochemical and morphometric study. Coll Antropol 2001;25(2):545-553.

25. Nobrega ACL, Freitas EV, Oliveira MAB, Leitão MB, Lazzoli JK, Nahas RM, et al. Position of the Brazilian Society of Sports Medicine and Brazilian Society of Geriatrics and Gerontology: Physical activity and health in the elderly. Rev Bras Med Esporte 2000;6(2):35-39.

26. Hughes VA, Frontera WR, Wood M, Evans WJ, Dallal GE, Roubenoff R, et al. Longitudinal muscle strength changes in older adults: influence of muscle mass, physical activity, end health. Journal of Gerontology 2001;56(5):B209-217.

27. Peterson DH, Jones GR, Rice CL. Ageing and physical activity: evidence to develop exercise recommendations for older adults. Can J Public Health 2007;98(2):S69-108.

\author{
Endereço para correspondência \\ José Rodrigo Pauli \\ Av. D. Ana Costa, 95 \\ Departamento de Biociências. Vila Mathias \\ 11060-001 - Santos, SP. Brasil \\ E-mail: rodrigosere@yahoo.com.br
}

